\title{
Starch phosphorylation associated SNPs found by genome-wide association studies in the potato (Solanum tuberosum L.)
}

Vadim K. Khlestkin ${ }^{1,2^{*}}$, Irina V. Rozanova ${ }^{1}$, Vadim M. Efimov ${ }^{1,3}$ and Elena K. Khlestkina ${ }^{1,3,4}$

From 11th International Multiconference "Bioinformatics of Genome Regulation and StructurelSystems Biology" - BGRSISB-

2018

Novosibirsk, Russia. 20-25 August 2018

\begin{abstract}
Background: The natural variation of starch phosphate content in potatoes has been previously reported. It is known that, in contrast to raw starch, commercially phosphorylated starch is more stable at high temperatures and shear rates and has higher water capacity. The genetic improvement of phosphate content in potato starch by selection or engineering would allow the production of phosphorylated starch in a natural, environmentally friendly way without chemicals. The aim of the current research is to identify genomic SNPs associated with starch phosphorylation by carrying out a genome-wide association study in potatoes.

Results: A total of $90 \mathrm{~S}$. tuberosum L. varieties were used for phenotyping and genotyping. The phosphorus content of starch in 90 potato cultivars was measured and then statistically analysed. Principal component analysis (PCA) revealed that the third and eighth principal components appeared to be sensitive to variation in phosphorus content ( $p=0.0005$ and $p=0.002$, respectively). PC3 showed the correlation of starch phosphorus content with allelic variations responsible for higher phosphorylation levels, found in four varieties. Similarly, PC8 indicated that hybrid 785/8-5 carried an allele associated with high phosphorus content, while the Impala and Red Scarlet varieties carried alleles for low phosphorus content. Genotyping was carried out using an Illumina $22 \mathrm{~K}$ SNP potato array. A total of 15,214 scorable SNPs (71.7\% success rate) was revealed. GWAS mapping plots were obtained using TASSEL based on several statistical models, including general linear models (GLMs), with and without accounting for population structure, as well as MLM. A total of 17 significant SNPs was identified for phosphorus content in potato starch, 14 of which are assigned to 8 genomic regions on chromosomes 1, 4, 5, 7, 8, 10, and 11. Most of the SNPs identified belong to protein coding regions; however, their allelic variation was not associated with changes in protein structure or function.
\end{abstract}

Conclusions: A total of 8 novel genomic regions possibly associated with starch phosphorylation on potato chromosomes 1, 4, 5, 7, 8, 10, and 11 was revealed. Further validation of the SNPs identified and the analysis of the surrounding genomic regions for candidate genes will allow better understanding of starch phosphorylation biochemistry. The most indicative SNPs may be useful for developing diagnostic markers to accelerate the breeding of potatoes with predetermined levels of starch phosphorylation.

\footnotetext{
* Correspondence: khlestkin@bionet.nsc.ru

${ }^{1}$ Institute of Cytology and Genetics, Siberian Branch of the Russian Academy

of Sciences, Lavrentjeva Ave. 10, Novosibirsk 630090, Russia

${ }^{2}$ Russian Research Institute of Farm Animal Genetics and Breeding - Branch

of the L.K. Ernst Federal Science Center for Animal Husbandry, St.

Peterburg-Tyarlevo, Moskovskoe shosse, 55a 196625, Russia

Full list of author information is available at the end of the article
}

(c) The Author(s). 2019 Open Access This article is distributed under the terms of the Creative Commons Attribution 4.0 International License (http://creativecommons.org/licenses/by/4.0/), which permits unrestricted use, distribution, and

reproduction in any medium, provided you give appropriate credit to the original author(s) and the source, provide a link to the Creative Commons license, and indicate if changes were made. The Creative Commons Public Domain Dedication waiver (http://creativecommons.org/publicdomain/zero/1.0/) applies to the data made available in this article, unless otherwise stated. 


\section{Background}

The potato is the third most important food crop in the world, and it has reliably held this position for centuries [1]. The plant was brought from Peru and Chile to Europe around the year 1570 [2] and reached Russia in the late seventeenth century [3]. The potato was used almost exclusively for food purposes in Russia until the very end of the nineteenth century, when potato starch became a standalone product. Since that time, potato starch has been used in both the food and technical industries. In the food industry, the potato is a common filler, gelling and texturizing agent, as well as a precursor for glucose, molasses, and dextrin production. Technical applications for starch are mainly in textile and paper manufacturing. Despite the fact that Russia is the world's third largest manufacturer of potatoes [4], it mainly imports potato starch due to the limited number of potatoes processed into raw starch. A negligible amount of the raw starch is further transformed into chemical and biochemical products [5]. Considering that potato tubers cannot be stored for long periods of time, the use of potatoes in the form of starch and products from starch modification is a good alternative. To increase the appeal of potato starch as a feedstock for various industries, substantial attempts to improve its molecular composition and physical and chemical properties are being made. The manipulation of certain gene networks may result in modified potato lines that produce starch with particular properties. This process has already been used to develop potato varieties with altered amylose and amylopectin content to produce starch that yields a clearer and more viscose gel, stable in freeze-thaw cycles [6].

Another feature of potato starch that could be improved with significant practical benefits is its phosphate content. Phosphate groups in potato starch are normally chemically bound to amylopectin molecules with approximately one phosphate group per 200-300 glucose units $(0.05-0.08 \% \mathrm{P})$. The negatively charged phosphate groups result in better dispersion of the polysaccharide chains in water due to mutual Coulomb repulsion. In industry, phosphorylated starch is manufactured in a chemical process that results in the addition of $1-3 \%$ phosphate content by mass. Commercially phosphorylated starch is known as food additive E1410 and functions as a texturizing and stabilizing agent. In contrast to raw starch, it is chemically more stable at high temperatures and shear rates and has a higher water capacity. It has been demonstrated that the physical and chemical properties of potato starch gels is dependent on its phosphate content [7]. Thus, genetically improving the phosphate content in potato starch through selection or genetic engineering would allow the production of phosphorylated starch in a natural, environmentally friendly way without the use of chemicals or other pollutants. Additionally, phosphorus is important for proper nutrition, and potatoes and starch high in phosphate would be valuable as food.

There have been some successful attempts to genetically modify potatoes that produce starch with a higher phosphate content. The expression of the laforin protein in the tuber [8] resulted in an average increase of 19\% in phosphate content with a simultaneously increase in the amount of amylopectin in the starch. A potato $\alpha$-glucan, water dikinase (GWD1) introduced into tubers of the amylose-containing line Kardal and the amylose-free mutant amf resulted in two contrasting effects; some plants showed higher phosphate content than the corresponding control, while others exhibited lower phosphate content, thereby generating starches with broad-scale variation in phosphate content [9]. The introduction of an (engineered) 4, 6- $\alpha$-glucanotransferase (GTFB) from Lactobacillus reuteri 121 into the same lines resulted in a significant increase in starch phosphate content in the amf line, while starches from the Kardal background did not show any changes in phosphate content [8].

It was shown that $\alpha$-glucan, water dikinase (GWD/ GWD1) and phosphoglucan, water dikinase (PWD/GWD3) enzymes play key roles in starch metabolism. Improved physical and chemical properties of starches isolated from GWD-deficient plants were described using Arabidopsis as a model [10].

In addition to reverse genetic approaches, the tools of forward genetics such as QTL analysis $[11,12]$ and association mapping [13] were used to reveal loci associated with starch phosphorylation in potatoes. The development of the $22 \mathrm{~K}$ SNP potato array with a high density of markers (on average, one SNP per $40 \mathrm{kbp}$ compared to one marker per $4 \mathrm{Mbp}$ in the abovementioned studies) allows us the opportunity to broaden the number of identified genomic loci associated with starch phosphorylation.

We performed a genome-wide association study using a $22 \mathrm{~K} \mathrm{SNP}$ potato array to find novel genomic regions associated with starch phosphorylation.

\section{Methods}

\section{Plant material}

The set of 90 potato (Solanum tuberosum L.) varieties from ICG "GenAgro" collection (Novosibirsk, Russia) was grown during the period May to October 2017 in the same field in Novosibirsk region (Michurinsky settlement, $54^{\circ} 52^{\prime} \mathrm{N}$ and $83^{\circ} 00^{\prime} \mathrm{E}$ ). A subset of 8 varieties was preliminary grown during the period May to October 2016 in the same field. Growing of potato plants was performed according to the standard procedure. Briefly, seed tubers of all cultivars were planted in two rows with $0.75 \mathrm{~m}$ spacing and $0.3 \mathrm{~m}$ distance between the plants on the rows. In total, 10 plants were planted in the row, so, the length of each row was $10 \mathrm{~m}$. Each cultivar was planted in three replicates, and distances between the 
replicates' plots were $2 \mathrm{~m}$. Sowing was performed in the first decade of May and harvesting in the 3rd decade of September.

After harvesting tubers were stored for 3 weeks at $+4{ }^{\circ} \mathrm{C}$. Only healthy tubers were collected for further analyses. From the healthy tubers, $25 \%$ of the smallest and largest tubers were removed. Among the rest, only five morphologically typical for a given variety tubers were selected for starch isolation.

\section{Starch isolation}

Potato starch was isolated form the tuber according to the typical procedure, described elsewhere (for example, see [14].

\section{DNA isolation and genotyping}

DNA was isolated from tubers skin using DNeasyPlant Mini Kit (Qiagen) according to the standard procedure. Concentration and purity of the isolated DNA were tested by gel electrophoresis and micro spectrophotometry on Nanodrop 2000 equipment.

All 90 accessions were genotyped using Illumina $22 \mathrm{~K}$ SNP potato array (GGP Potato V3) at the Traitgenetics $\mathrm{GmbH}$ (Gatersleben, Germany). The Illumina Infinium procedure was performed according to the manufacturer's protocol. None of the samples were failed for the analysis.

\section{Phosphorus content analysis}

Analysis of the starch samples was performed using phosphomolibdate method with spectrophotometric detection according to the standard procedure, described in GOST 7698-93 "Starch. Rules for acceptance and methods of analysis" (Russian and Belarus standard, correlates with ISO 3946-82). In brief, starch probe was decomposed in a mixture of sulfuric and nitric acids, neutralized and reacted with ammonium molibdate. Spectrophotometric detection at $825 \mathrm{~nm}$ allowed to determine content of phosphorus in the initial starch probe with the help of the calibration curve.

\section{PCA and population structure analysis}

The Principal component analysis (PCA) was considered with genotyping data calculated through the distance matrix. It was done using software packages STATISTICA 8 [15]. In order to calculate the population structure matrix (Q-matrix) containing membership coefficients for each individual of potato mapping panel the genotyping data was analyzed by Bayesian cluster analysis in STRUCTURE v.2.3.4 [16]. The number of population was taken as $\mathrm{k}=3$, which corresponded to number of clusters according to STATISTICA.

\section{Association analysis}

Different statistical models were tested on disease resistance scores (separately for each of four isolates) with the help of TASSEL 5 package [17] to detect significant marker associations: (1) general linear model (GLM) without taking into account population structure, (2) GLM with using a Q-matrix of population membership (GLM) taking into account the population structure, (3) GLM with taking into account population membership estimates derived from principal components analysis (GLM + PCA), and (4) a composite approach that combines both Q-matrix and the average relationship between individuals or lines (null matrix) represented in TASSEL as a linear model (MLM).

Since TASSEL have specially been developed for diploid genome analysis, we re-coded tetraploid potato genome from four-letter code to numerical, taking into account the dose of certain allele. After the re-coding, 0 was assigned to effector allele and 1 - as non-effector allele, and their intermediate forms were coded as 0.75 , 0.5 and 0.25 . For example, AAAA allele is reflected as 1 , AAAG - as 0.75, AAGG - as 0.5, AGGG - as 0.25, and GGGG - as 0 .

To identify significant SNPs two corrections were used: (i) the Bonferroni correction, where the significant threshold (0.05) is divided by the total number of tests, in this case, the total number of markers $(27,319)$, giving threshold $1.8302 * 10^{-6}$, and (ii) the false discovered rate (FDR) that was calculated for each isolate in each model. Percentage of random was $<10 \%$.

\section{Results and discussion}

\section{Phenotyping, genotyping and population structure}

Initially, the starch phosphorus content was determined for 8 varieties of potato harvested in 2016 and 2017. A strong positive correlation of this trait between 2 years (Pearson's coefficient 0.91) was found in most of the cultivars (Fig. 1). The correlation of high phosphorus content in the crops for both years indicated that genetics rather than the environment contributed to the variation among the genotypes for starch phosphorylation level. Thus, to perform the association mapping, the starch phosphorus content was determined for 90 varieties of potato harvested in 2017.

The SNP-genotyping of the 90 varieties harvested in 2017 was performed on an Illumina $22 \mathrm{~K}$ potato array (GGP Potato V3) and revealed a set of 15, 214 (71.7\%) scorable SNPs. SNPs were treated as significant only if they were discovered in $>95 \%$ of the studied genotypes. We used the chromosomal position of the SNPs [18] to arrange the marker dataset on the corresponding chromosomes with the help of a specially written script. The average coverage was one SNP per $40 \mathrm{kbp}$ calculated from the known length of the potato genome (n), which 


\section{Correlation of $\mathrm{P}$ content}

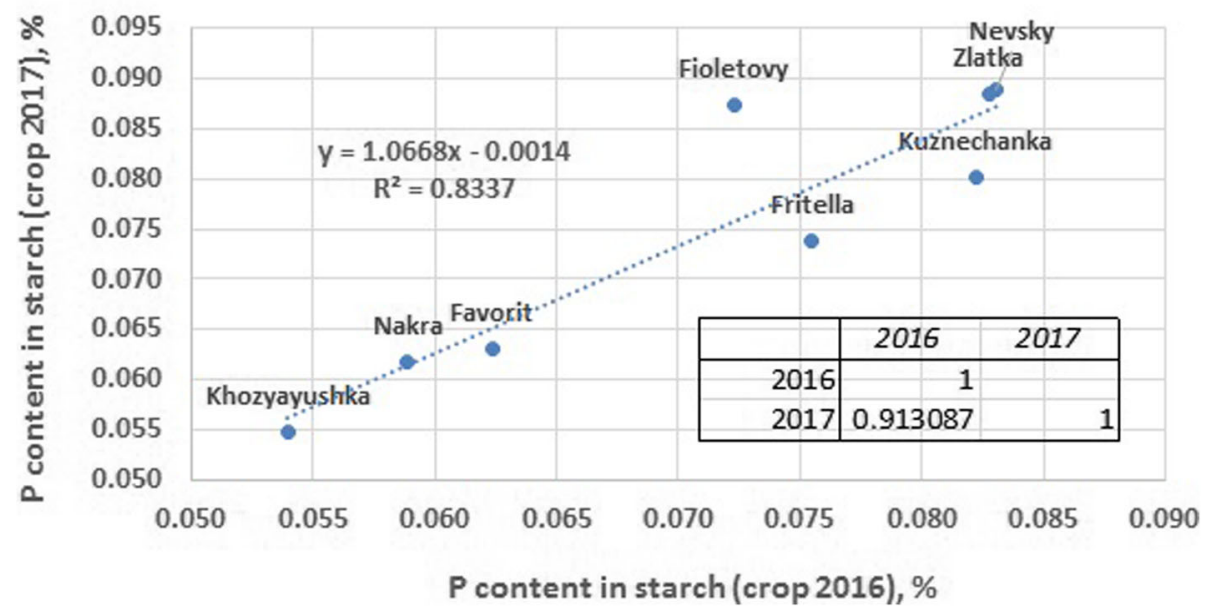

Fig. 1 A strong positive correlation of starch phosphorylation in the tubers of potato varieties harvested in 2016 and 2017

is approximately $840 \mathrm{Mbp}$ [19]. Previous association studies and QTL analyses for phosphorus content in potato tubers were based on a restricted number of markers with an average of 1 marker per $4 \mathrm{Mbp}$ [11-13].

Population structure analysis showed that for the set of 90 potato varieties, a total of 23 principal components contributed more than $1 \%$ of common genotype variance. The first three components described $31.8 \%$ of variance. Two dimensional projections of the first three principal components using PC1-PC2, PC2-PC3 and PC1-PC3 (Fig. 2) uncovered three clusters, highlighted in green ellipses. The projection of PC1-3 also revealed that there are extreme differences between some varieties in the study set.

Only the third and eighth principal components ( $p=0.0005$ and $p=0.002$, respectively) appeared to account for phosphorus content. Correlation of the starch phosphorus content with PC3 suggested that the Ladozhsky, Nevsky, Ruslan and Kuznechanka varieties carry alleles responsible for higher phosphorus content (Additional file 1). Similarly, the correlation with PC8 suggested variety $785 / 8-5$ carried an allele pattern associated with high phosphorus content, and the Impala and Red Scarlet varieties carried alleles for low phosphorus content (Additional file 1). Figure 3 reflects the correlations in the third and eighth principal components. Varieties with high and low phosphorous content are marked with green and red ellipses, respectively.

\section{Association mapping}

The association-mapping plots were obtained with the TASSEL program by using the SNP-genotyping and phenotyping results for all 90 varieties. QQ- and Manhattan plots shown in Additional files 2 and 3, respectively, were obtained from the application of different statistical methods to the genotyping and phenotyping correlation results. The basic approach used GLM without considering population structure. To build on this, GLM was used with a Q-matrix of population membership, which takes into account population structure. Population membership estimates serve as covariates in the model and can be derived using principal component analysis (PCA) [20]. For each marker-trait combination, GLM finds the ordinary least squares solution as described previously [21]. The model can include main effects, interactions, nested effects and covariates. A composite approach that combines both the Q-matrix and the average relationship between individuals or lines (null matrix) represented in TASSEL as a linear model (MLM) function has been shown to be superior [22] to GLM methods. Finally, we used all 4 approaches: (1) general linear model (GLM) without considering population structure, (2) GLM with a Q-matrix of population membership (GLM) taking into account population structure, (3) GLM taking into account population membership estimates derived from principal components analysis (GLM + PCA), and (4) a composite approach that combines both the Q-matrix and the average relationship between individuals or lines (null matrix) represented in TASSEL as a linear model (MLM) (Additional file 3). A total of 17 significant possible SNPs was found (Table 1). SNPs with a $p$-value that overcame either $3.29 \mathrm{e}^{-6}$ (Bonferroni level) or the less strict FDR (false discovery rate) criteria were considered. The Bonferroni correction sets very strict criteria; overcoming it implies the greatest likelihood of SNP-trait association, but permits false negative results, omitting some trait-associated SNPs [23]. To counteract this, the less strict FDR criteria were also applied. Three of the 17 SNPs identified were not assigned to a chromosome (Table 1). The rest of the 14 markers were assigned to 

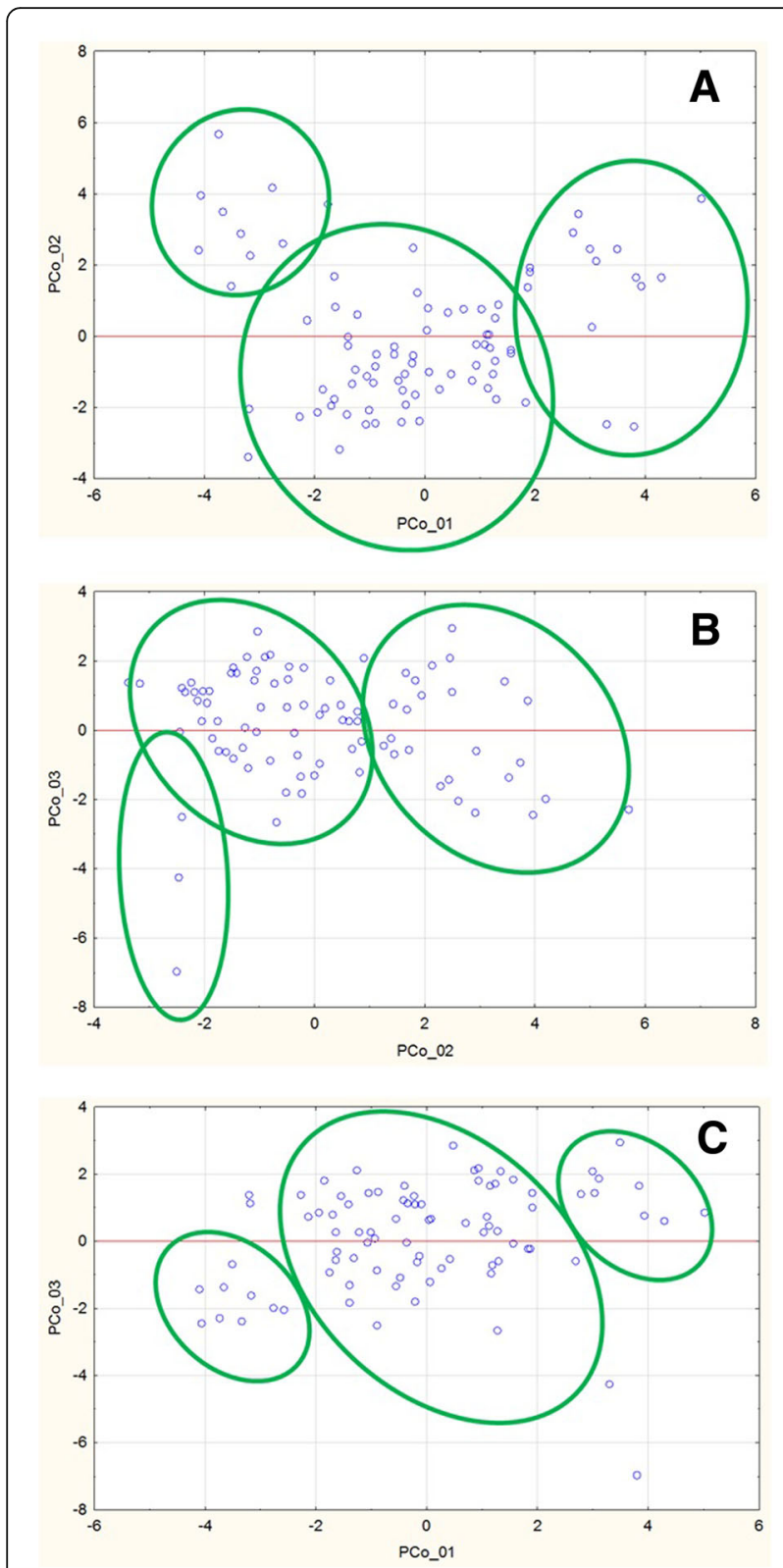

Fig. 2 Three clusters of the potato population studied based on SNP-genotyping data. a Principal components $1-2 ;$ b principal components 2-3; c principal components $1-3$

chromosomes 1, 4, 5, 7, 8, 10 and 11. Twelve of these 14 SNPs are located in protein-coding regions (Table 2).

\section{Chromosome 1}

Six significant SNPs were found on chromosome 1 (Table 1). Five of the six markers were mapped closely to each other, corresponding to one genomic region approximately $50 \mathrm{kbp}$ in length (between $69,872,835$ and $69,918,804$ bp of chromosome 1). The SNPs were located in protein-coding regions of genes: $1 \mathrm{SNP}$ was in a gene encoding carotenoid cleavage dioxygenase 7; 2 SNPs were located in a gene encoding a mitochondrial elongation factor; and 2 SNPs were in a malate dehydrogenase gene (Table 2). Allelic variation in these SNP loci is to due synonymous substitutions only.

Another significant SNP was located about $10 \mathrm{Mbp}$ from the genomic region described above (Table 1). The SNP was found in the gene PGSC0003DMG402018257 coding for a leucine-rich, repeat-containing protein (Table 2). Allelic variation in this SNP locus results in an amino acid substitution, $\mathrm{Val}<=>\mathrm{Il}$, though does not change the main properties of the amino acid residue, since both valine and isoleucine are aliphatic hydrophobic amino acids. An association between potato chromosome 1 and the phosphorus content of potato starch has never before been reported. Further investigation of the two genomic regions identified on chromosome 1 is needed to find and validate candidate genes associated with phosphorus content variation in potato tubers.

\section{Chromosome 4}

A single significant SNP was revealed on chromosome 4 (Table 1). The SNP was in a gene encoding a maltose transporter. Allelic variation in this SNP locus does not result in an amino acid change (Table 2). No QTL has previously been mapped to chromosome 4 for phosphorus content. However, Carpenter et al. [13] reported a relationship between the starch branching enzyme I gene $(S B E I)$ and starch phosphorylation. The SBEI location is $4: 71586223-71,597,347$, which is approximately 13 Mbp distal from the PotVar0084444 SNP, suggesting that the phosphorus content-associated region identified on chromosome 4 in the current study is not related to SBEI.

\section{Chromosome 5}

Two significant SNPs were found on chromosome 5 (Table 1). These SNPs were found in the non-coding regions of the chromosome. They are approximately 2.8 Mbp from each other. Previously, a QTL for phosphorus content related to potato starch was reported for this locus [11, 12]. Werij et al. [12] suggested that this locus was related to the GWD (GWD1) gene encoding $\alpha$-glucan, water dikinase (syn.: Starch-granule-bound R1 protein). Carpenter et al. [13] also observed an association between the GWD gene and the phosphorus content in tuber starch. The GWD gene location is 5:9823451-9,838,970. The distance between this gene and the region found in the current study is greater than $30 \mathrm{Mbp}$.

\section{Chromosome 7}

Potato chromosome 7 has never before been reported to be associated with phosphorus content in tuber starch. Here, the significant chromosomal region was found to be associated with the solcap_snp_c2_38828 marker (Table 1). The SNP was located in the gene PGSC0003DMG400011132 


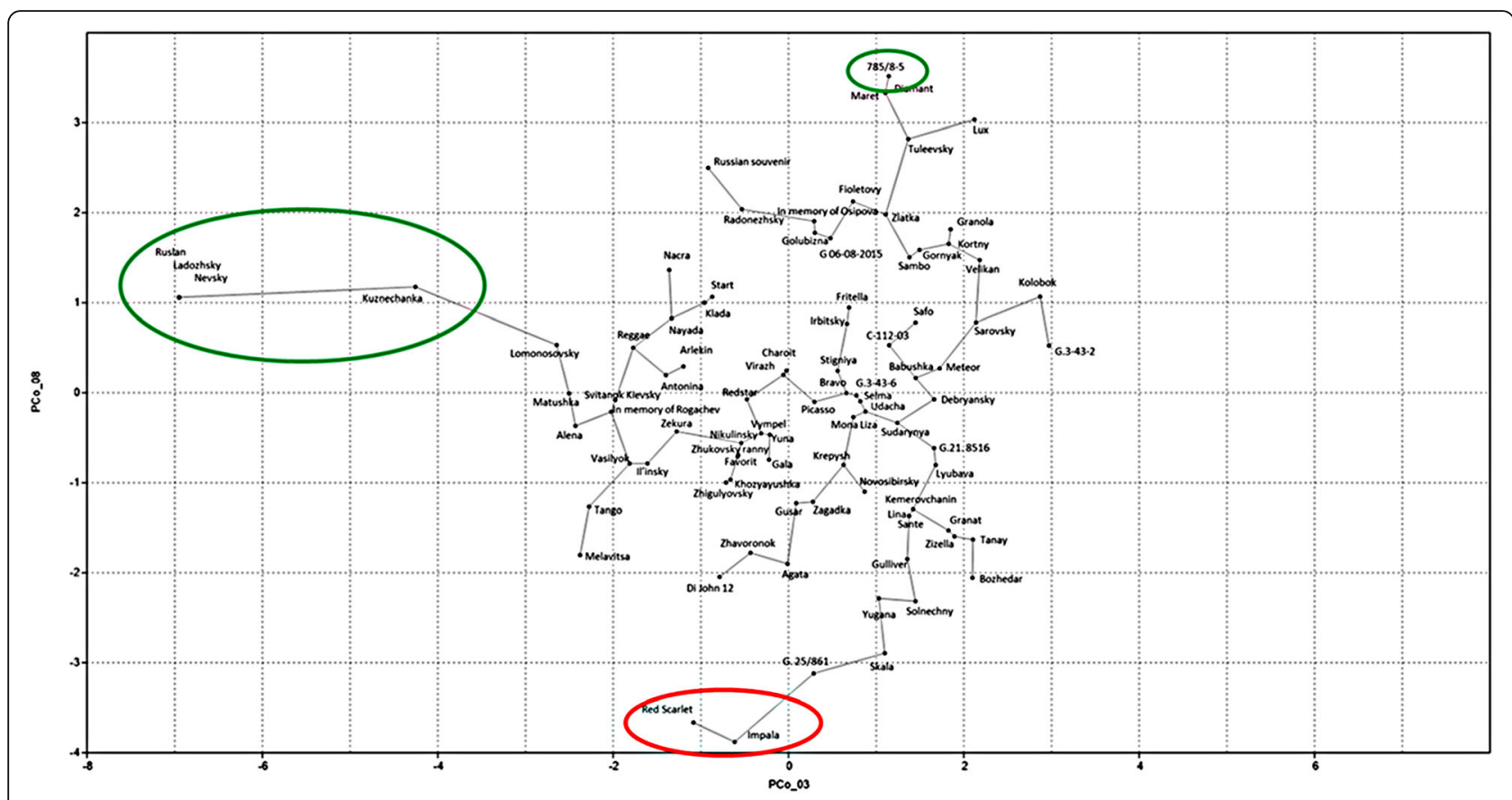

Fig. 3 Correlation of the third and eighth principal components. Varieties with high and low phosphorous content are marked with green and red ellipses, respectively

Table 1 Significant SNPs discovered with the four statistical models

\begin{tabular}{|c|c|c|c|c|c|c|c|c|}
\hline No & Marker & Chromosome & Position & Correction & $p$-value & $\begin{array}{l}\text { Minor allele } \\
\text { frequency }\end{array}$ & Polymorphism & $\begin{array}{l}\text { Allele associated with high } \\
\text { phosphorylation level }\end{array}$ \\
\hline 1 & PotVar0043426 & 1 & $69,895,805$ & Bonferroni & 0,00000064 & 0,35 & $A / G$ & G \\
\hline 2 & solcap_snp_c2_17529 & 1 & $69,902,011$ & Bonferroni & 0,00000131 & 0,32 & $A / G$ & G \\
\hline 3 & solcap_snp_c1_5759 & 1 & $69,917,893$ & Bonferroni & 0,00000167 & 0,40 & $A / G$ & G \\
\hline 4 & solcap_snp_c2_17530a & 1 & $69,918,804$ & Bonferroni & 0,00000104 & 0,33 & T/A & A \\
\hline 5 & PotVar0043516 & 1 & $69,872,835$ & Bonferroni & 0,00000303 & 0,38 & $A / G$ & G \\
\hline 6 & solcap_snp_c1_2519 & 1 & $79,487,269$ & Bonferroni & 0,00000332 & 0,21 & $\mathrm{~T} / \mathrm{C}$ & C \\
\hline 7 & PotVar0084444 & 4 & $58,263,204$ & FDR & 0,0000887 & 0,04 & $\mathrm{~T} / \mathrm{C}$ & C \\
\hline 8 & PotVar0018043 & 5 & $33,966,509$ & FDR & 0,0000165 & 0,24 & $A / G$ & G \\
\hline 9 & solcap_snp_c2_50231 b & 5 & $36,834,630$ & FDR & 0,000027 & 0,33 & $\mathrm{~T} / \mathrm{C}$ & T \\
\hline 10 & solcap_snp_c2_38828 b & 7 & 588,137 & FDR & 0,0000375 & 0,21 & $A / G$ & G \\
\hline 11 & solcap_snp_c1_6252 & 8 & $20,465,382$ & FDR & 0,0000661 & 0,05 & $A / G$ & A \\
\hline 12 & PotVar0065847 & 10 & 134,951 & FDR & 0,0000245 & 0,13 & $A / G$ & G \\
\hline 13 & PotVar0065745 & 10 & 197,180 & FDR & 0,0000339 & 0,13 & $A / G$ & G \\
\hline 14 & solcap_snp_c1_2187 ${ }^{b}$ & 11 & $2,562,328$ & FDR & 0,000036 & 0,46 & $A / G$ & G \\
\hline 15 & solcap_snp_c2_55899 & Unknown & Unknown & FDR & 0,00000901 & 0,30 & $\mathrm{~T} / \mathrm{G}$ & T \\
\hline 16 & solcap_snp_c2_53177 & Unknown & Unknown & FDR & 0,0000373 & 0,05 & $\mathrm{~T} / \mathrm{C}$ & T \\
\hline 17 & solcap_snp_c2_7785 ${ }^{\mathrm{d}}$ & Unknown & Unknown & FDR & 0,0000663 & 0,07 & $\mathrm{~A} / \mathrm{G}$ & G \\
\hline
\end{tabular}




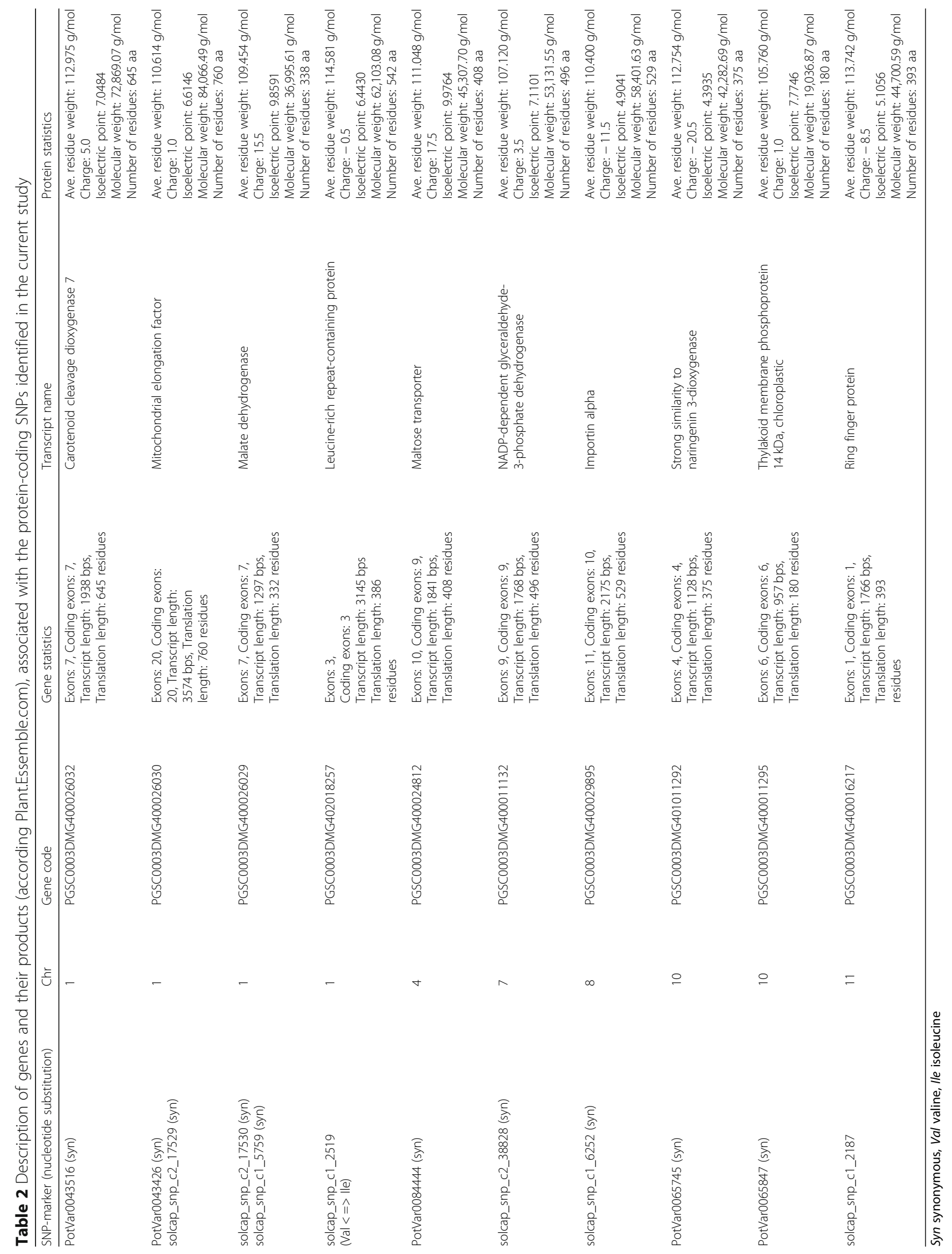


encoding a NADP-dependent glyceraldehyde-3-phosphate dehydrogenase protein. Allelic variation in this SNP locus does not result in an amino acid substitution (Table 2). Further investigation of this gene, comparisons of allelic differences in coding regions between varieties with high and low phosphorus content as well as application of reverse genetics tools are needed to check whether this gene contributes to phosphorylation levels in potato tuber starch.

\section{Chromosome 8}

The association between potato chromosome 8 and starch phosphorylation has not been reported previously. A significant SNP was found on chromosome 8 (Table 1). The SNP was located in the gene coding region for an importin alpha protein. Nucleotide changes in this SNP locus are synonymous (Table 2). The product of this gene is responsible for alpha protein import - a large class of proteins, which may include enzymes responsible for phosphorylation. Without detailed experimental investigation of this gene and neighbouring genes in the chromosomal region identified in the current study, it is not possible to assume a candidate gene.

\section{Chromosome 10}

Potato chromosome 10 has never been reported to be associated with phosphorus content in tuber starch before. Two significant SNPs were revealed in the current study (Table 1). These SNPs were in protein-coding genes, one having strong similarity to naringenin 3-dioxygenase (PGSC0003DMG401011292) and the other to thylakoid membrane phosphoprotein (PGSC0003DMG400011295). They are situated approximately $63 \mathrm{kbp}$ from each other. Naringenin 3-dioxygenase participates in biosynthesis of secondary phenolic metabolites and is most likely unrelated to starch phosphorylation. Thus, significance of the SNP in PGSC0003DMG401011292 may be explained by its close linkage to PGSC0003DMG400011295 or other genes affecting phosphorus content. Substitution in the SNP found in the gene PGSC0003DMG400011295 is synonymous. Further investigation of this gene and neighbouring genes is needed.

\section{Chromosome 11}

A single significant SNP was located on chromosome 11 (Table 1). It was found in a gene encoding a ring finger protein. Allelic variation in this SNP locus does not result in an amino acid change (Table 2). This is the first report of a region associated with starch phosphorylation on chromosome 11 and is of some interest; however, without further investigation of this region, it is difficult to make any conclusions about possible candidate genes.

In addition to the listed potato chromosomes above, the 2nd and 9th chromosomes are associated with tuber starch phosphorylation [11-13]. By sequencing amplicons and comparing this with phenotypic data, putative candidate genes involved in starch metabolism were genotyped [13], and markers associated with C6-phosphorylation (GWD gene, chromosome 5), C3-phosphorylation (PWD/ GWD3 gene, chromosome 9), and phosphorylation in both positions (SBEI - chromosome 4, SBEII - 9, SSII - 2, and SSIII - 2) were uncovered (chromosome assignment of the genes is given according to Plant.Essemble.com or taken from a review [6]). Carreno-Quintero, et al. [11] revealed QTLs on chromosomes 2, 5, and 9 and reported the correlation of starch phosphorylation with seven metabolites ( $\beta$-Ala, GABA, L-Asp, Ala, butanoic acid, and two unknown compounds). QTLs for these metabolites colocalize with at least one of the starch phosphorylation QTLs. Starch phosphorylation QTLs were found and mapped on chromosomes 2, 5 and 9 by Werij et al. [12]. These QTLs colocalize with SSII on chromosome 2, GWD on chromosome 5, and StPho2 on chromosome 9 [12].

\section{Conclusions}

A genome-wide association study using a $22 \mathrm{~K} \mathrm{SNP} \mathrm{po-}$ tato array allowed 8 novel genomic regions on chromosomes $1,4,5,7,8,10$, and 11 associated with starch phosphorylation to be found. Some of the SNPs identified were located in non-coding genomic regions. Allelic variation in the SNPs found in protein coding regions was not related to changes in protein structure or function. Further validation of the SNPs identified and the analysis of the surrounding genomic regions for candidate genes will allow a better understanding of starch phosphorylation biochemistry. Among the genes carrying significant SNPs identified in the current study, the gene on chromosome 7 encoding a NADP-dependent glyceraldehyde-3-phosphate dehydrogenase protein is a primary target for a reverse genetic investigation of a potential functional association with tuber starch phosphorus content.

\section{Additional files}

Additional file 1: Scatterplots of phosphorus content in starch of potato varieties analyzed, showing sensitivity of the third (A) and eighth (B) components to phosphorus content. The blue ellipses show varieties, which high or low phosphorous contents in starch are genetically determined. (PDF $314 \mathrm{~kb}$ )

Additional file 2: QQ-plots (quantile-quantile plots) for the models: (1) GLM without correction for population structure; (2) GLM + Q: GLM + Q-matrix to account for population structure; (3) GLM + PCA, (4) MLM. (PDF 282 kb)

Additional file 3: Association mapping results for phosphorous content scorings in potato starch using different models: GLM without accounting for population structure, GLM + Q (GLM + Q-matrix to account for population structure), GLM + PCA, and MLM. Dash line named "Bonferroni" corresponds the Bonferroni threshold. Dash line named "FDR" corresponds the FDR (false discovered rate) threshold. Vertical axes show the -log (10) of marker trait association $p$-value. Chr1-12 - chromosome assignment of loci. ChrO - unassigned loci. (PDF 787 kb) 


\section{Acknowledgments}

We thank ICG collection "GenAgro" (Novosibirsk, Russia) providing potato plant collection and personally Anna Safonova. We also thank Tatiana Erst and Dr. Igor Totsky, participating in sample preparation, as well as Dr. Pavel Barsukov, contributing to phenotyping, and, finally, the Traitgenetics $\mathrm{GmbH}$ (Gatersleben, Germany) for providing service on genotyping of potato cultivars using $22 \mathrm{~K}$ Illumina SNP array.

\section{Funding}

Publication costs were funded by the Russian Foundation for Basic Research (No 17-29-08006). Growth of potato plants in the ICG Plant Growth Core Facility was supported by ICG project 0324-2018-0018.

\section{Availability of data and materials}

Not applicable.

\section{About this supplement}

This article has been published as part of BMC Genetics Volume 20 Supplement 1, 2019: Selected articles from BGRSISB-2018: genetics. The full contents of the supplement are available online at https:/bmcgenet.biomedcentral.com/ articles/supplements/volume-20-supplement-1.

\section{Authors' contributions}

VKK initiated the study, contributed to its design and conception, performed population structure analysis, participated in phenotyping, and drafted the manuscript. IVR performed PCA and GWAS analyses. VME participated in statistical analysis of genotyping and phenotyping data. EKK contributed to interpretation of data and to revising the manuscript critically. All authors read and approved the final manuscript.

\section{Ethics approval and consent to participate}

Not applicable.

\section{Consent for publication}

Not applicable.

\section{Competing interests}

The authors declare that they have no competing interests.

\section{Publisher's Note}

Springer Nature remains neutral with regard to jurisdictional claims in published maps and institutional affiliations.

\section{Author details}

${ }^{1}$ Institute of Cytology and Genetics, Siberian Branch of the Russian Academy of Sciences, Lavrentjeva Ave. 10, Novosibirsk 630090, Russia. ${ }^{2}$ Russian Research Institute of Farm Animal Genetics and Breeding - Branch of the L.K. Ernst Federal Science Center for Animal Husbandry, St. Peterburg-Tyarlevo, Moskovskoe shosse, 55a 196625, Russia. ${ }^{3}$ Novosibirsk State University, Pirogova Str., 1, Novosibirsk 630090, Russia. ${ }^{4}$ N.I. Vavilov All-Russian Research Institute of Plant Genetic Resources (VIR), Bolshaya Morskaya Str., 42-44, St. Petersburg 190000, Russia.

\section{Published: 18 March 2019}

\section{References}

1. Spooner DM, Ghislain M, Simon R, Jansky SH, Gavrilenko T. Systematics, diversity, genetics, and evolution of wild and cultivated potatoes. Bot Rev. 2014;80:283-383. https://doi.org/10.1007/s12229-014-9146-y.

2. Grommers HE, van der Krogt DA. Potato Starch: Production, modifications and uses. In: Miller J, Whistler R, editors. Starch chemistry and technology. 3rd Edn. Elsevier Inc; 2009. p. 511-39. ISBN: 978-0-12-746275-2.

3. Simakov EA, Yashina IM, Sklyarova NP. Potato breeding in Russia: history, general trends and achievements. In: Haverkort AJ, Anisimov BV, editors. Potato production and innovative technologies. The Netherlands: Wageningen Academic Publishers; 2007. p. 311-27.

4. Vassilieva Y. Overview of Potato Supply and Demand in Russia. Report of USDA Foreign Agricultural Service. 2013. https://www.google.com/url?sa=t\&rct=j\&q= \&esrc $=$ \&\&source $=$ web\& $\& d=1 \&$ ved $=2$ ahUKEwjwutramNLgAhWGwMQBHbv8 ALUQFjAAegQ|AxAC\&url=https\%3A\%2F\%2Fgain.fas.usda.gov\%2FRecent\% 2520GAIN\%2520Publications\%2FOverview\%2520of\%2520Potato
\%2520Supply\%2520and\%2520Demand\%2520in\%2520Russia_Moscow_Russian \%2520Federation_10-31-2013.pdf\&usg=AOVVaw2IWfi2zoNQbWIIUh92oqWN. Accessed 3 Sept 2018.

5. Khlestkin V, Peltek S, Kolchanov N. Review of direct chemical and biochemical transformations of starch. Carbohydr Polym. 2018;181:460-76. https://doi.org/10.1016/j.carbpol.2017.10.035.

6. Khlestkin V, Peltek S, Kolchanov N. Target genes for development of potato (Solanum tuberosum L.) cultivars with desired starch properties. Sel'skokhozyaistvennaya Biologiya [Agricultural Biology]. 2017;52(1):25-36. https://doi.org/10.15389/agrobiology.2017.1.25eng.

7. Hemar Y, Hardacre A, Hedderley DI, Clark S, Illingworth D, Harper JW, Boland M. Relationship between the pasting behaviour and the phosphorus content of different Potato starches. Starch. 2007;59:149-55.

8. Xu X, Dechesne A, Visser RGF, Trindade LM. Expression of an (engineered) 4,6-a-Glucanotransferase in Potato results in changes in Starch characteristics. PLoS One. 2016;11(12):e0166981. https://doi.org/10.1371/ journal.pone.0166981.

9. Xu X, Dees D, Dechesne A, Huang X-F, Visser RGF, Trindade LM. Starch phosphorylation plays an important role in starch biosynthesis. Carbohydr Polym. 2017;157:1628-37. https://doi.org/10.1016/j.carbpol.2016.11.043.

10. Mahlow S, Hejazi M, Kuhnert F, Garz A, Brust H, Baumann O, Fettke J. Phosphorylation of transitory starch by a-glucan, water dikinase during starch turnover affects the surface properties and morphology of starch granules. New Phytol. 2014;203:495-507. https://doi.org/10.1111/nph.12801.

11. Carreno-Quintero N, Acharjee A, Maliepaard C, Bachem CWB, Mumm R, Bouwmeester H, Visser RGF, Keurentjes JJB. Untargeted metabolic quantitative trait loci analyses reveal a relationship between primary metabolism and Potato tuber quality. Plant Physiol. 2012;158:1306-18. https://doi.org/10.1104/pp.111.188441

12. Werij JS, Furrer $H$, van Eck HJ, Visser RGF, CWB B. A limited set of starch related genes explain several interrelated traits in potato. Euphytica. 2012; 186:501-16. https://doi.org/10.1007/s10681-012-0651-y.

13. Carpenter MA, Joyce NI, Genet RA, Cooper RD, Murray SR, Noble AD, Butler RC, Timmerman-Vaughan GM. Starch phosphorylation in potato tubers is influenced by allelic variation in the genes encoding glucan water dikinase, starch branching enzymes I and II, and starch synthase III. Front Plant Sci. 2015;6:143. https://doi.org/10.3389/fpls.2015.00143.eCollection 2015.

14. Chung H-J, Li X-Q, Kalinga D, Lim S-T, Yada R, Liu Q. Physicochemical properties of dry matter and isolated starch from potatoes grown in different locations in Canada. Food Res Int. 2014;57:89-94. https://doi.org/ 10.1016/j.foodres.2014.01.034.

15. Weiß CH. Statsoft, inc., tulsa, ok.: Statistica, version 8; 2007.

16. Pritchard JK, Stephens M, Donnelly P. Inference of population structure using multilocus genotype data. Genet. 2000;155:945-59.

17. Bradbury PJ, Zhang Z, Kroon DE, Casstevens TM, Ramdoss Y, Buckler ES. TASSEL: software for association mapping of complex traits in diverse samples. Bioinformatics. 2007;23:2633-5.

18. Vos PG, Uitdewilligen JGAML, Voorrips RE, Visser RGF, van Eck HJ. Development and analysis of a 20K SNP array for potato (Solanum tuberosum): an insight into the breeding history. Theor Appl Genet. 2015; 128:2387-401. https://doi.org/10.1007/s00122-015-2593-y.

19. Visser RGF, Bachem CWB, de Boer JM, Bryan GJ, Chakrabati SK, Feingold S, Gromadka R, van Ham RCHJ, Huang S, Jacobs JME, Kuznetsov B, de Melo PE, Milbourne D, Orjeda G, Sagredo B, Tang X. Sequencing the potato genome: outline and first results to come from the elucidation of the sequence of the world's third most important food crop. Am J Potato Res. 2009;86:417-29. https://doi.org/10.1007/s12230-009-9097-8.

20. Zhao K, Aranzana MJ, Kim S, Lister C, Shindo C, Tang C, Toomajian C, Zheng H, Dean C, Marjoram P, Nordborg M. An Arabidopsis example of association mapping in structured samples. PLoS Genet. 2007;3(1):e4.

21. Searle SR. Linear models for unbalanced data. New York: Wiley; 1987. p. 533.

22. Yu JM, Pressoir G, Briggs WH, Bi IV, Yamasaki M, Doebley JF, McMullen MD, Gaut BS, Nielsen DM, Holland JB, Kresovich S, Buckler ES. A unified mixedmodel method for association mapping that accounts for multiple levels of relatedness. Nat Genet. 2006;38:203-8.

23. JH MD. Handbook of biological statistics. 2nd ed. Baltimore: Sparky House: 2011. p. 257. 